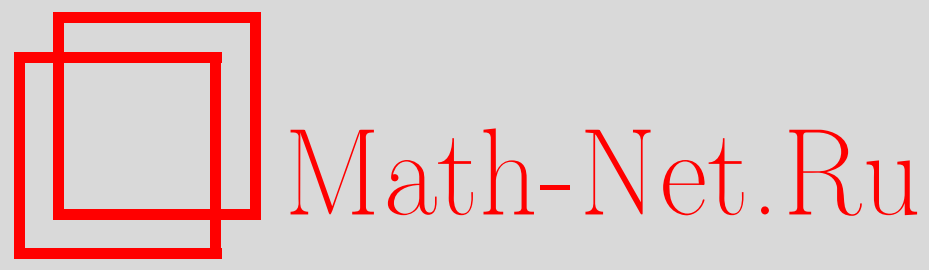

В. Л. Левин, Двойственные представления выпуклых тел и их поляр, Функи. анализ и его прил., 1996, том 30, выпуск 3, 79-81

DOI: https://doi.org/10.4213/faa533

Использование Общероссийского математического портала Math$\mathrm{Net.Ru}$ подразумевает, что вы прочитали и согласны с пользовательским соглашением

http://www . mathnet.ru/rus/agreement

Параметры загрузки:

IP : 107.22 .136 .117

26 апреля 2023 г., 15:20:16

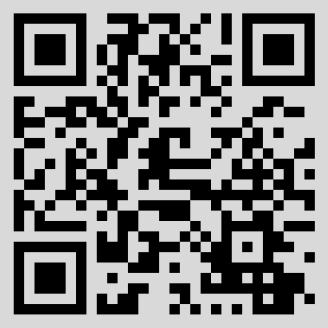


Спроектируем грани $\Gamma_{1}, \ldots, \Gamma_{n-1}$ вдоль вектора $v_{n}$ на содержащую точку нуль гиперплоскость $L_{\Gamma}$, параллельную грани $Г$. Ориентируем $L_{\Gamma}$ в соответствии с ориентацией грани $\Gamma$.

По предположению индукции в $(n-1)$-мерном пространстве формула справедлива. Применим ее для набора сдвинутых в гиперплоскость $L_{\Gamma}$ многогранников $\Gamma_{1}, \ldots, \Gamma_{n-1}$. Тогда из [2] и леммы 1 следует, что формула справедлива и в $n$-мерном пространстве в случае, когда векторы $v_{n}$ трансверсальны соответствующим плоскостям $L_{\Gamma}$.

В заключение я хочу поблагодарить Б. Я. Казарновского и А. Г. Хованского за полезные обсуждения.

\title{
ЛИтЕРАТУРА
}

1. Гельфонд О. А., Хованский А. Г. Вычеты Гротендика и многогранники Ньютона. Докл. РАН (в печати). 2. Бураго Д. М., Залгаллер В. А. Геометрические неравенства. Наука, Л., 1980.

Институт системных исследований РАН

Поступило в редакцию 25 декабря 1995 г.

УдК 513.88

\section{Двойственные представления выпуклых тел и их поляр}

\author{
(c) 1996. В. Л. ЛЕвин
}

1. Везде далее $E$ - вещественное отделимое локально выпуклое пространство и $E^{*}$ - сопряженное к нему пространство. Выпуклым телом называется такое замкнутое выпуклое множество $C$ в $E$, что $C \neq E$ и $0-$ его внутренняя точка. Множество граничных точек множества $C$ обозначается через $b(C)$.

ОПРЕДЕЛЕНИЕ 1 . Пусть $C$ - выпуклое тело в $E, x \in b(C)$ и $x^{\prime} \in E^{*} \backslash\{0\}$. Замкнутая гиперплоскость

$$
H\left(x, x^{\prime}\right):=\left\{y \in E:\left\langle y, x^{\prime}\right\rangle=\left\langle x, x^{\prime}\right\rangle\right\}
$$

называется опорной к $C$ в точке $x$, если $C$ лежит в полупространстве

$$
H^{\leqslant}\left(x, x^{\prime}\right):=\left\{y \in E:\left\langle y, x^{\prime}\right\rangle \leqslant\left\langle x, x^{\prime}\right\rangle\right\}
$$

или, что равносильно, если $x^{\prime} \in \mathscr{N}_{C}(x)$, где

$$
\mathscr{N}_{C}(x):=\left\{x^{\prime} \in E^{*}:\left\langle y-x, x^{\prime}\right\rangle \leqslant 0 \quad \forall y \in C\right\}
$$

- нормальный конус множества $C$ в $x$. Будем считать, не ограничивая общности, что $x^{\prime}$ нормирован условием $\left\langle x, x^{\prime}\right\rangle=1$.

ОПРЕДЕЛЕНИЕ 2. Опорная гиперплоскость $H\left(x, x^{\prime}\right)$ называется касательной $\kappa C$ в $x$, если $x^{\prime}$ - единственный элемент из $\mathscr{N}_{C}(x)$, такой, что $\left\langle x, x^{\prime}\right\rangle=1$. В этом случае $x$ называется точкой гладкости множества $b(C)$.

Касательная гиперплоскость и соответствующее касательное полупространство в $x$ обозначаются далее через $H(x)$ и $H^{\leqslant}(x)$, а множество точек гладкости множества $b(C)$ - через $\Gamma(C)$. 
Напомним (см., например, [1]), что полярой множества $C$ называется множество

$$
C^{0}:=\left\{x^{\prime} \in E^{*}:\left\langle x, x^{\prime}\right\rangle \leqslant 1 \quad \forall x \in C\right\} .
$$

Точка $x_{0}^{\prime} \in C^{0}$ называется слабо* выступающей, если для некоторого $y \in E$ справедливо неравенство

$$
\left\langle y, x^{\prime}\right\rangle<\left\langle y, x_{0}^{\prime}\right\rangle \quad \forall x^{\prime} \in C^{0} \backslash\left\{x_{0}^{\prime}\right\} .
$$

В таком случае говорят, что $y$ выделяет точку $x_{0}^{\prime}$. Множество $\exp C^{0}$ слабо* выступающих точек множества $C^{0}$ содержится в множестве крайних точек этого множества, но в отличие от последнего может оказаться пустым (см. пример ниже).

ТЕОРема 1 (теорема двойственности). Следуюшие утверждения о выпуклом теле $C$ равносильньи:

(1) справедливо представление

$$
C=\bigcap_{x \in \Gamma(C)} H^{\leqslant}(x) ;
$$

(2) справедливо представление

$$
C^{0}=\operatorname{cl} \operatorname{co} \exp C^{0},
$$

где $\mathrm{cl}$ со обозначает слабо* замкнутую выпуклую оболочку.

Теорема 2. Если $\Gamma(C)$ плотно в $b(C)$, то имеют место представления (*) $u(* *)$.

Выделим основные этапы доказательства теоремы 1 в виде трех лемм.

Лемма 1. Пусть $\Gamma \subseteq b(C)$ u $\xi: \Gamma \rightarrow E^{*}$ - отображение, такое, ито $\xi(x) \in \mathscr{N}_{C}(x)$ u $\langle x, \xi(x)\rangle=1$ для любой точки $x \in \Gamma$. Следуюшие утверждения равносильнь:

(1) имеет место представление

$$
C=\bigcap_{x \in \Gamma} H^{\leqslant}(x, \xi(x)) ;
$$

(2) имеет место представление $C^{0}=\operatorname{cl} \operatorname{co}(0 \cup \xi(\Gamma))$.

ЛЕмма 2 (в случае банахова пространства ср. [2, предложение 6.9]). Пусть $x^{\prime} \in C^{0} \backslash\{0\}$. Следующие утверждения равносильньи:

(a) $x^{\prime} \in \exp C^{0}$

(б) существует точка $x \in \Gamma(C)$, такал, ито $\left\langle x, x^{\prime}\right\rangle=1$ u $H(x)=H\left(x, x^{\prime}\right)$.

Лемма 3. Если $C^{0}=\operatorname{cl} \operatorname{co}\left\{0 \cup\left(\exp C^{0} \backslash\{0\}\right)\right\} \quad u 0 \notin \operatorname{cl} \operatorname{co}\left(\exp C^{0} \backslash\{0\}\right), m o$ $0 \in \exp C^{0}$.

Теорема 2 вытекает из теоремы 1 и следующей леммы.

Лемма 4. Пусть $C$ - выпуклое тело, $\Gamma$ - множество, плотное в $b(C)$, $u \xi: \Gamma \rightarrow E^{*} \backslash\{0\}$ - отображение, удовлетворяющее условию $\xi(x) \in \mathscr{N}_{C}(x)$ для любой точки $x \in \Gamma$. Тогда имеет место (***).

2. Пусть теперь $E$ - нормированное пространство. Напомним (см. [2]), что банахово пространство $E$ входит в класс $G D S$ пространств со свойством дифференцируемости по Гато, если для каждого открытого выпуклого множества 
$U \subseteq E$ и каждой непрерывной выпуклой функции на $U$ точки, где эта функция дифференцируема по Гато, плотны в $U$. Класс $G D S$ содержит рефлексивные пространства, сепарабельные пространства, пространства с гладкой нормой и пространства, сопряженные к которым допускают строго выпуклую эквивалентную норму [2]. Из соединения теорем 1 и 2 с известными характеризациями класса $G D S$ (см. [2, теорема 6.2 и следствие 6.6]) вытекает следующая

ТЕОРЕМА ДВОЙСТВЕННОСТИ В МАССОВОЙ ПОСТАНОВКЕ. Пусть $E-\sigma a-$ нахово пространство. Следующие утверждения равносильнь:

(1) $E \in G D S$;

(2) для каждого выпуклого тела $C \subset E$ множество $\Gamma(C)$ плотно в $b(C)$;

(3) каждое выпуклое тело $C \subset E$ допускает представление (*);

(4) поляра $C^{0}$ каждого выпуклого тела $C \subset E$ допускает представление $(* *)$.

ЗАМЕЧАНИЕ. Остается открытым вопрос о том, является ли для фиксированного $C \subset E$ плотность множества $\Gamma(C)$ в $b(C)$ необходимым условием справедливости представлений $(*)$ и $(* *)$.

В заключение приведем пример выпуклого тела $C$ в сепарабельном нормированном пространстве, для которого множества $\Gamma(C)$ и $\exp C^{0}$ пусты и, стало быть, представления $(*)$ и $(* *)$ не имеют места.

Пример. $E$ - нормированное подпространство в $l^{1}$, состоящее из финитных последовательностей, т. е. $x=\left(x_{n}\right) \in E \Longleftrightarrow x_{n}=0 \forall n>n_{0}(x)$, и $C$ единичный шар в $E$. Имеем $E^{*}=l^{\infty}$ и для каждого $x \in b(C)$ любой элемент $x^{\prime}=\left(x_{n}^{\prime}\right) \in l^{\infty}$ с $x_{n}^{\prime}=\operatorname{sgn}\left(x_{n}\right)$ при $n \in \operatorname{supp} x=\left\{k: x_{k} \neq 0\right\}$ и $\left|x_{n}^{\prime}\right| \leqslant 1$ при $n \notin \operatorname{supp} x$ содержится в конусе $\mathscr{N}_{C}(x)$. Так как таких $x^{\prime}$ бесконечное множество и для каждого из них $\left\langle x, x^{\prime}\right\rangle=1$, то $\Gamma(C)$ пусто. Далее, $C^{0}$ есть единичный шар в $l^{\infty}$, и так как элементы пространства $E$ (финитные последовательности) не выделяют точек шара $C^{0}$, то $\exp C^{0}$ тоже пусто.

ЗАмечАниЕ. Аналогичное рассуждение показывает, что $\Gamma(C)=\varnothing$ и $\exp C^{0}$ $=\varnothing$, где $C$ - единичный шар банахова пространства $l^{1}(J)$ функций $x: J \rightarrow$ $\mathbb{R}$ на несчетном бесконечном множестве $J$, для каждой из которых $\operatorname{supp} x=$ $\{j \in J: x(j) \neq 0\}$ не более чем счетно и

$$
\|x\|:=\sum_{j \in \operatorname{supp} x}|x(j)|<+\infty .
$$

Можно также привести пример выпуклого тела $C$, для которого $Г(C)$ и $\exp C^{0}$ непусты, но представления $(*)$ и $(* *)$ не имеют места.

\section{ЛИТЕРАТУРА}

1. Бурбаки H. Топологические векторные пространства. ИЛ., М., 1959. 2. Phelps R. R. Convex Functions, Monotone Operators and Differentiability. SpringerVerlag, 1989.

Центральный экономико-математический институт РАH

Поступило в редакцию

6 апреля 1995 г.

В переработанном виде 31 октября 1995 г. 